Z Herz- Thorax- Gefäßchir 2018 · 32:433

https://doi.org/10.1007/s00398-018-0281-3

(c) Springer Medizin Verlag GmbH, ein Teil von Springer Nature 2018

CrossMark

\author{
A. Welz \\ Halblech, Deutschland
}

\section{Neues und Bewährtes}

Liebe Leserinnen und Leser,

diese Ausgabe der Zeitschrift für Herz-, Thorax- und Gefäßchirurgie weist mit über 90 Druckseiten einen außergewöhnlichen Umfang auf. Dies ist der Tatsache geschuldet, dass die Herausgeber sich entschlossen haben, 3 Übersichtsarbeiten zur Thematik der katheterbasierten Klappenchirurgie in einem Heft zusammenzufassen. Während die Übersichtsarbeit von Lars Conzelmann sehr detailliert technische Aspekte der Transkatheter-Aortenklappenimplantation darstellt, geben die Arbeiten von Miriam Silaschi und Lenard Conradi umfassende Einblicke in die heute bereits erreichten Möglichkeiten der Transkatheter-Aortenklappen-, aber auch Transkatheter-Mitralklappenimplantation. Darüber hinaus befasst sich ein Beitrag in der Rubrik "Aus der DGTHG“ mit der Problematik der Indikationsstellung und Patientenselektion für die kathetergestützte Behandlung der Mitralklappenimplantation (Empfehlungen unserer Arbeitsgruppe „kathetertechnische Herzklappenchirurgie“).

\section{》) Kardiovaskuläre Chirurgie ohne endovaskuläre Verfahren wird in naher Zukunft nicht mehr denkbar sein}

Ich bin überzeugt, dass das vorliegende Heft in der Lage sein wird, die rasanten Fortschritte moderner kathetertechnischer Verfahren sowie deren zukünftig steigende Bedeutung kenntlich $\mathrm{zu}$ machen und insbesondere unsere in Ausbildung befindlichen jüngeren Kollegen ermuntern wird, „wire skills“ zu erwerben. Kardiovaskuläre Chirurgie ohne endovaskuläre Verfahren wird bereits in naher Zukunft nicht mehr denkbar sein.

Zusätzlich möchte ich auf den Beitrag in der Rubrik „CME Zertifizierte Fortbildung" hinweisen. Auf dem Gebiet der Koronarchirurgie kann die Herzchirurgie auf eine lange zurückgehende Erfolgsgeschichte verweisen. Nach Einführung der Bypass-Chirurgie in das Spektrum kardiochirurgischer Operationen durch Favaloro vor nun über 50 Jahren wurde das Verfahren in mehreren Studien rasch einer randomisierten Prüfung unterzogen. Auch neuere Studien bewiesen die Überlegenheit dieses Verfahrens bei komplexeren Formen koronarer Pathologien. Dies hat wiederum prominent Eingang in die in diesem Jahr aktualisierten Leitlinien der European Society of Cardiology (ESC)/European Association for Cardio-Thoracic Surgery (EACTS) zur Revaskularisation bei stabiler koronarer Herzkrankheit gefunden. So erhielt die koronare Bypassoperation mit Ausnahme der Ein- und Zweigefäßerkrankung ohne proximale Beteiligung der „Left anterior descending (LAD) artery" in allen übrigen Fällen eine Klasse-1-Empfehlung mit Evidenzgrad A.

Ich erlaube mir, an dieser Stelle zu betonen, dass sich die Therapieprinzipien der interventionellen und chirurgischen Behandlung insoweit wesentlich unterscheiden, als die interventionelle Therapie immer im Läsionsbereich agiert, während der Bypass in der Regel in der Lage ist, die pathologisch veränderten proximalen Bezirke zu überspringen. Somit wird die Therapie der koronaren Herzkrankheit mit PCI und Stent wohl immer durch Prä-, In- und Post-Stent-Rezidive belastet sein. Dies umso mehr, als pathophysiologisch lange bekannt ist, dass die koronare Herzkrankheit in vielen Fällen dominant am proximalen Gefäßbereich entsteht, während der chirurgisch angelegte Bypass die pathologisch weniger belasteten distalen Gefäßprovinzen nutzt. Hier Stimmen in exzellenter Weise theoretische Überlegungen und empirische Daten, also deduktiver und induktiver wissenschaftlicher Ansatz, überein.

Die Autoren der weiteren ebenso sorgfältig ausgearbeiteten sowie spannenden Arbeiten unserer sonstigen Rubriken mögen mir diesen ausführlicheren Ausflug in die kathetergestützte Chirurgie und Koronarchirurgie verzeihen.

Abschließend wünsche ich Ihnen, liebe Leser, viel Spaß und Erkenntnisgewinn beim Durcharbeiten dieser Ausgabe der Zeitschrift für Herz-, Thorax- und Gefäßchirurgie.

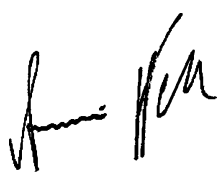

Prof. Dr. med. Armin Welz

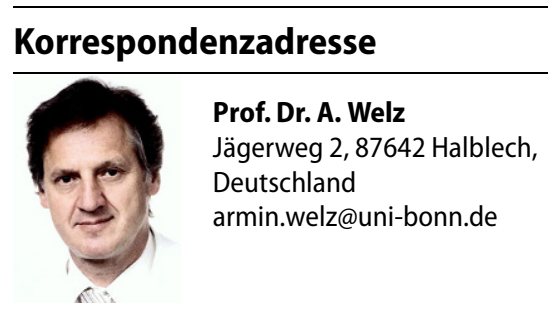

Interessenkonflikt. A. Welz gibt an, dass kein Interessenkonflikt besteht. 